JURNAL AL BAYAN: JURNAL JURUSAN PENDIDIKAN BAHASA ARAB

p-ISSN 2086-9282 | e-ISSN 2549-1229

\title{
Musykilat al-Tarbiyyah Tuwajihuha al-Thalabah fi Ta'allum al-Lughah al-Arabiyah li Ghairi al-Nathiqin biha
}

\author{
Himatul Istiqomah ${ }^{1}$, Hanik Mahliatussikah ${ }^{2 *}$ \\ ${ }^{1}$ Arabic Education Study Program, Universitas Negeri Malang, Indonesia \\ ${ }^{2}$ Arabic Education Study Program, Universitas Negeri Malang, Indonesia
}

Article History:

Received : September 08, 2019

Revised : October 15, 2019

Accepted : November 11, 2019

Published : December 01, 2019

Keywords:

Arabic; Eksternal; Internal; Learning; Linguistic

*Correspondence Address:

hanik.mahliatussikah.fs@um.ac.id

\begin{abstract}
The rapid development of technology is expected to develop the teaching and learning of Arabic. However, due to some problems, this aim is yet to achieve. This descriptive qualitative research is to identify problems faced by Indonesian learners of Arabic and to explore the types of problems and the solutions. Based on a library research, there are two main problems in teaching and learning of Arabic. They are internal and external problems as well as linguistic and non-linguistic problems. Internal problems are related to learners while external problems are related to environment and teachers. Linguistic problems deal with sound system, vocabulary, semantics, syntax, morphology, and writing. Nonlinguistic problems are those dealing with school facility, family, and learners' internal factors. Learners facing linguistic problems should be given exercises to improve their linguistic competence and those facing non-linguistic problems should be given motivation, feedback, and helped to view the process of Arabic learning as something fun. Families should consider the most appropriate school for their children. Schools, on the other hand, are responsible to give the best facilities such as experienced teachers and learning facilities that are able to boost learners' language learning, as well as giving weak learners additional materials to improve their ability. This discussion is expected to reveal the factors causing the problems and identify their possible solutions. Thus, this research is significant as it contributes to solve problems in non-Arab learning of Arabic
\end{abstract}

$$
\text { أكثر من كانت اللغة العربية إحدى اللغات الرسمية الست في منظمة الأمم المتحدة، يتحدثها }
$$


حتى يكون تعليمها وتعلمها يتجاه إلى إتقان المهارات الاتصالية بها.بالرغم من ذلك، ليس تعليم اللغة العربية للناطقين بغيرها وتعلمها أمرين سهلين. بل لا تجرّد منهما المشكلات المتنوعة. وقد قال عبد الرحمن (1989) إن المشكلة هي الصعوبة المحسوسة للفرد وبين تحقيق أكبر قدر ممكن من التوافق في أي المجال. فطبعا أن مع المشكلة حلّا، كما أن مع العسر يسرا. ولكن، لن تصل المشكلة إلى حلّها تامّة. وأثناء عملية الاكتشاف للطريقة اللائقة تظهر المشكلة مجدّدا. والمشكلة الجديدة تتعلق بالمشكلة السابقة. وهكذا، هناك العلاقة بين المشكلة السابقة والتالية وعكسها.

التربية لها المعاني الكثيرة عند العلماء وفقا على خلفياتهم المختلفة. لقد ذهب الفلاسفة وعلماء الأخلاق أن التربية هي القيام بالتغييرات في الفرد جسميا وروحيا إلى أعلى درجات الجمال والكمال. ولقد ذهب علماء النفس والسلوك أن التربية هي السعي لتنمية القوى الذهنية والأعمال السلوكية. ولقد ذهب علماء الاجتماع أنها انتقال ثروة الحضارة للوصول إلى الأهداف الاجتماعية. ولقد ذهب مناظر الإسلام أن التربية إحياء وإشـاد فطرة الله في الإنسان من خلال الحركة وتنمية قدرته للوصول إلى الكمال اللامتناهي.2 ومن المعاني السابقة نستخلص بأن التربية هي عملية تساعد الإنسان للبلوغ إلى استقلال الشخصوتحقيق السلوك المفيد. هذا تنفيذ حديث رسول الله محمد صلى الله عليه وسلم، الذي رواه الترمذي من الصحابة أبي هريرة أنه قال: أكمل المؤمنين إيمانا أحسنهم خلقا.

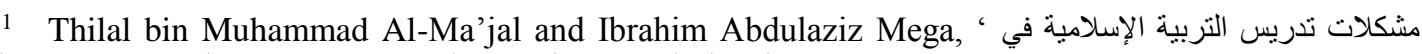
'المرحلة المنوسطة بالمدارس العربية الإسلامية في مالي من وجهة نظر المعلمين ', IUGJEPS, 25.2 (2017), $82-99$ (p. 85). 
ومن الدراسات السـابقة حول مشكلات التربية، فهناك عدة الخبرات ومنها المشكلات

تواجهها الطلبة في جامعة الجوف بالقريات هي مشتملة على المشكلات السيكولوجية، والأكاديمية،، والاجتماعية.33 و قد ذكر السبياني (2016)Al-Subyani أن المشكلات تواجهها الطلبة في جامعة الملك عبد العزيز الكبرى مشتملة على المشكلات الإدارية، والاقتصيادية، ثم الأكاديمية.4 إن المشكلات تواجهها الطلبة في جامعة أولوداغ مدينة بورصة تركيا عند دويغون وغوليك (2012)Doygun \& Gullec مشتملة على التكيف والتغذياة، والملائمة والنزعة، واضطراب عن الاستقبال والبطالة، والتربية المؤهلة. 5 وقد ذكرت خفيفة وأخواتها Khofifah, et al (2017) أن المشكلات المواجهاة عند الطلبة هي مشكلات الفرد والتعلم والاجتماعي والمهنة. 6 وروحانسيا Ruhansih) قالت إن المشكلات تواجهها الطلبة في المدرسة هي مشكلات التعلم المؤثرة بعدم الحفز للتعلم، وبعامل البيئة اللامؤيَّدة على حالة التعلم، وبحالة الأبواين من جهة الاجتماعي والاقتصادي، وطلاق الأبوين. وقد ذكرت شـافيني وأخواتها Syafini, et al (2013) أن مشكلات التعلم تواجهها الطلبة هي المهارة لتنظيم

2 Ali Al-Qaimi, أسس التربية (Beirut: Daar el-Nubala, 1995), pp. 25-29.

Abeer Hameed Al-Bashtawi, 'Student's Perspectives for the Encountered Problems \& Challenges at the Department of English in The Colege of Sciences \& Arts In Alqurrayyat', International Journal of Education and Research, 2.2 (2014), 1-8.

4 Noor Abdulhadi Al-Subyani, 'The Academic, Administrative, Economic, Social, and Psychological Problems Faced by Students of Textile and Clothing Major at King Abdul-Aziz University', International Education Studies, 2016, 155-62 (p. 155).

5 Ozlem Doygun and Selma Gulec, 'The Problem Faced by University Students and Proposal for Solution', 2012, XLVII, 1115-23.

6 Aulia Khofifah, Afrizal Sano, and Yarmis Syukur, 'Permasalahan yang Disampaikan Siswa kepada Guru BK/Konselor', Jurnal Educatio; Jurnal Pendidikan Indonesia, 3.1 (2017), 45-52.

7 Dea Siti Ruhansih, 'Masalah-Masalah Siswa Di Sekolah Serta Pendekatan-Pendekatan Umum Dalam Bimbingan Dan Konseling Ditinjau Dari "Kacamata Konselor (Studi Deskriptif Di SMP Nugraha Bandung)', Jurnal Quanta, 2.1 (2018), 1-3. 
وقت التعلم، ولقراءة الكتب، ولحفظ الدروس، ولمتابعة الدراسة في الفصل، وللكتابة، ولتلخيص الكتب، وللتعلم المجموعي، ولتذكر الدروس والتركيز عند التعلم، ولأداء

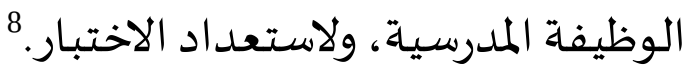

وكان برمادي Permadi) قد بين أن المشكلات تواجهها الطلبة في تخطيط

المهنة هي عدم التفاؤل، الذي يؤدي على صعوبة إنتاج الرأي لعلاج المشكلة وصعوبة الانضباط،ونقصان أوسع المعرفة عن الصنعة، وصعوبة نيل المعرفة عن المهنة اللائقة للعمل بعد الخروج في المدرساة، وضعف المهارات الاتصالية باللغة الأجنبية لأداء المقابلة العملية، وصعوبة تنظيم جدول الأنشطة المساعدة لتخطيط المهنة، وصعوبة أداء الوظيفة الجودية في تمام الوقت. وبيّن العلوامي والكوري (2016) عن المشكلات تواجهها الطلبة المعلمين في جامعة العين للعلوم والتكنولوجيا أثناء ممارستهم للتربية العملية الميدانية. وهي على الترتيب تشتمل على المشكلات التنظيمية لبرنامج التربية العملية، والمشكلات المتعلقة بإدارة المدرسة المتعاونة، ومشكلات الإشراف والتقويم، والمشكلات التدريسية والصفية. إن أعلى المشكلات تواجهها الطلبة المعلمين في جامعة مصراتة هو ارتباك الطالب المعلم عند دخول المشرف لحضيور الحصية. وأن أدناها هو تشدد معلم المادة في توجيهات

8 Elgi Syafini, Yarmis Syukur, and Indra Ibrahim, 'Masalah Belajar Siswa dan Penanganannya', Konselor; Jurnal Ilmiah Konseling, 2.2 (2013), 15-19.

9 Nofianti Eka Permadi, 'Masalah-Masalah yang Dihadai Peserta Didik dalam Perencanaan Karir dan Implikasinya Terhadap Pelayanan Bimbingan Karir’, Jurnal Penelitian Bimbingan dan Konseling, 2, 1 (2016), 134-45.

10 Abdullah Ali Al-Awami and Ahmad Yahya Al-Kuri, ' المشكلات التي يو اجهها طلبة الدبلوم المهني بجامعة العين للعلوم و التكنولوجيا في التربية العملية الميدانية., Journal of Al-Nasser University, 8 (2016), 196-225. 
الطلبة المعلمين.11 وقد ذكر العنزي (2015) أن المشكلات تواجهها الطلبة المعلمين في جامعة شقراء أكثرها مشكلات إدارية، ثم طبيعة البرنامج وطلبة المدرسـة.12 والسلخي (2010) قالت إن المشكلات تواجهها الطالبات المعلمات في جامعة البترا الخاصة هي المشكلات المتعلقة بالمشرف، وبالمعلمة المتعاونة، وبإدارة المدرسة المتعاونة، وبالطالبة المعلمة، وبالإمكانات المادياة.13 إن المشكلات تواجهها الطلبة المعلمين في الجامعة الهاشمية هي المشكلات المتعلقة بالروضة المتعاونة، وبرنامج التربية العملية، وشخصية الطالب المعلم، والإشراف على التربية العملية، والمعلمة المتعاونة، وتخطيط وتنفيذ الدروس.14 فجميع أنواع المشكلات السابقة هي المشكلات في مجال التربية عامة. و هذا البحث سيركز في مشكلات تعليم اللغة العربية لغير الناطقين بها حسب الدراسـة المكتبية في البحوث العلمية المنشورة وعن معالجتها. وعسى أن تكون نتائج هذا البحث مفيدة في مساعدة حلول مشكلات تعليم اللغة العربية. فمعرفة المشكلات ومعالجتها تسهل من يعلّم اللغة العربية لغير الناطقين بها، ومن يتعلمها في معرفة نوعية المشكلة تواجهها الطلبة ومعالجتها. إن الطبيب لا يستطيع أن يعطي أي دواء إلى المرضى إلا بعد معرفة المرض المصيب إليه وفحصيه.

11 Hasan Salim Asyuhubi and Ibrahim Otsman Arhim, ‘ المشكلات التي تواجه الطلبة المعلمين أثناء ممارستهم للتربية العملية من وجهةنظرالطلبة أنفسهم ', Journal of Education Faculty Misurata University, 5 (2016), 184-208.

12 Saud Farhan Al-Anzi, ' المشكلات التي تواجه طلبة التربية العملية في جامعة شقراء من وجهة نظر الطلبة المعلمين أنفسهم ', Journal of University of Babylon for Humanities, 23 (2015), 3-21.

13 Mahmoed Gamal Gamil Asalakhi, 'مشكلات التربية العملية من جهة نظر الطالبات المعلمات في جامعة البترا الخاصة), Majalah Mutah li al-Buhuts wa al-Dirasat: Silsilah al-Ulum al-Insaniyah wa al-Ijtima'iyah, 25.7 (2010), 199-234. 
الإطار النظري

مشكلات التربية تواجهها الطلبة

إن الأساس في هذه العقبات هو عدم وفاء الحاجات إلى الأمن داخل النفس

وخارجها. كان للطلبة الأمن الكافي داخل النفس، وهم لا يذمّون ويتأسّفون عيوبهم فقط.

فيستطيعوا أن يقوموا حياتهم بدوام الشكر على فضل الربّ تعالى بسائر مزاياهم

المفضيَّلة. حتى تكون حياتهم حياة طيبة ومؤهَّلة. و الأمن الكافي خارج النفس يكون ممن

أقرب إليهم خاصة من أمهاتهم أو أباتهم،وهذا يكفي كالزاد والحفز لهم ليقوموا حياتهم

الحسنى والمؤهلة. لأن الأبوين القوة والطاقة الثانية لدى الطلبة جانب قوتهم الأساسية

داخل نفسهم. إذا كان ثنائي القوة هذا تكافؤا، فأي المشكلات المواجهة عند الطلبة ستنتهي فور|15. - n.

والمثال كما قد كتب أندرسون أن العوامل الجغرافية أيدرجة الحرارة في كل

الاختلافات الموسيمية تؤثر على تطوير عملية التربية خاصة في بعض الدول في أوروبا الغربيةة'. والمثال البسيط لهذه العوامل إذ كانت المدرسة قريبة منالسكّة

14 Mushthofa Fankhur Khuwalda, Fathi Mahmoed Ahameeda, and Saad Abdul Qadir Al-Hijazi, 'مشكلات التربية العملية التي تو اجه الطلبة المعلمين في تخصص تربية طفل في كلية الملكة رانيا للطفولة بالجامعة الهاشمية، Journal of Damascus University, 26.3 (2010), 737-81.

15 Himatul Istiqomah and Halimi, 'اتجاهات كون النبي والرسول في شخصية محمد في نثر مولد البرزنجي', Arabi: Journal of Arabic Studies, 1, 2 (2017), 127-40 (pp. 132-33).

16 D Anderson, 'Geographic and Economic Factors and Developmet of Educational System in Western Europe', The University of Chicago Journals, 9.2 (1965), 147-54 (p. 153). 
الحديدياة،والمطار، والمحطة، أو الوسائل العامة الأخرى التي تؤدي إلى الجلبة. كل الجلبة منها يزعج تركيز الطلبة أثناء الدراسة.17.

والعقبات المتعلقة بالنواقص ونقاط الضعف. فيه مذكور أن العقبات تتكون من

نقص المعلمين والقوى البشرية، عدم توفر برنامج معين مدروس، نقص البيانات، نقص

التجهيزات، ميزانية التربية الباهظة، النواقص ذات العلاقة بالأسلوب التعليهي،

الاتجاهات القديماة، الاتجاه الظني، الفقر، ضعف الإيمان، ضعف الآداب والقيود، فراغ

وعبثية المناهج، ضعف الإدارة، عدم المسؤولية، انتشار الجرائم والفساد، هبوط

المستوى العلمي، للعالمين في الحقل الثقافي، نقص إمكانيات المطبوعات الجيدة، التركيز

على الجوانب النظرية في المدارس، عدم القبول بالنقد، ضعف واهتزاز الأسس العلمية. وكلها تعتبر كالموانع في عملية التربية¹8.

المشكلات اللغوية وغير اللغوية

تنقسم المشكلات في تعليم اللغة العربية لغير الناطقين بها إلـقسمين 19.الأولى،

المشكلات اللغوية أي المشكلاتالمتعلقة بالأمور اللغوية مباشرة. وهي المشكلات عن الأصوات العربية، والمفردات، وقواعد اللغة العربية، وتراكيب اللغة العربية 20.وقد فصل الأعظمي أن المشكلات اللغوية المواجهة لدى الطلبة في تعلم اللغة اعربية لغير الناطقين

\footnotetext{
${ }^{17}$ Ismah and Erna Ratna Wibiastuti, 'Pengaruh Letak Geografis Sekolah Terhadap Konsentrasi Belajar Matematika Sekolah Menengah Pertama', FIBONACCI: Jurnal Pendidikan Matematika \& Matematika, 1.1 (2015), 82-96 (p. 93).

${ }^{18}$ Al-Qaimi, pp. 388-89.

${ }^{19}$ Aziz Fahrurrozi, 'Pembelajaran Bahasa Arab : Problematika Dan Solusinya', ARABIYAT: Jurnal Pendidikan Bahasa Arab Dan Kebahasaaraban, 1.2 (2014), 161-80 (p. 162) <https://doi.org/10.15408/a.v1i2.1137>.

${ }^{20}$ Fahrurrozi, pp. 162-64.
} 
بها عديدة. وهي المشكلات في القراءة وفهم المسموع والكتابة والتحدث واستخدام المعاجم اللغوية وفي المنهج2. والثانية، المشكلات غير اللغوية أي المشكلات خارج اللغة التي تؤثر تأثيرا قوياإلى نجاح عملية تعليم اللغة العربية لغير الناطقين بها. ومنها: الدافعة والإرادة، والوسائل التعليمياة، وكفاءة المعلمين، ومنهج التعليم، والحصة التعليمية، والبيئة اللغوية 22. والمهم فالأهم في نجاح عملية تعليم اللغة العربية لغير الناطقين بها هو وجود الدافعة والإرادة القوية والمتناسقة لدى الطلبة والمعلمين. ثم يتبعها بمنهج التعليم المتناسب بالأهداف التعليمية. حلول مشكلات التربية

وحسب مشكلات التربية أو عقبات التربية السابقة، فكما يلي الحلول لمعالجها،

$$
\text { 1. وهي حسب كل المجال والنوع من المشكلات. }
$$

قال القائمي أن الفرد والمجتمع يؤثران في نمو وتربية الفرد. دورهما مهم وعظيم. وعلى كل شخص أن يشعر بوظيفته تجاه الآخر. وأن عليه بناءه ورفح الموانع التربوية من أمامه، والعمل على تنميته وتوعيته، ويكون بمثابة عصا يتكأ عليها23.وأن مقتضى طبع الإنسان هو طلب النمو، والحقيقة، والسمو، والتحرك باتجاه الرغبات. فيمكن

${ }^{21}$ Majelis el-Nuwab el-Iraqi, 'قراءة في صعوبات تعلم مادة اللغة العربية في العراق', in الدورة الانتخابية الثانية (Iraq: Dairah el-Buhuts, 2014), pp. 7-8.

${ }^{22}$ Fahrurrozi, pp. 164-65.

${ }^{23}$ Al-Qaimi, p. 392. 
التقدم عن طريق إيجاد النضج وأرضية الوعي، وإزالة الموانع بالتدريج. هذا هو العمل يلاحظ في طريقة التربية للأنبياء 2. حلول المشكلات المتعلقة بالطلبة أنفسهم. المعالجة للمشكلات المتعلقة بالطلبة أنفسهم بالطرق المعينة، مثلما ذكر الغزالي في كتابه إحياء علوم الدين؛ يقوم المتعلم بتزكية النفس؛ والهجرة إلى المنظقة الجديدة لنمو شخصية التأهُب؛ يعلم عيوبه للتبعيد عن سوء السلوك مع زملائه مثل الكبر والأناني؛ ويعلم أهمية العلوم في الحياة.25. 3. حلول المشكلات المتعلقة بالأسـرة.

ومن المعالجات لهذه المشكلة هياختيار الوالدين المدرسة اللائقة للأبناء، ويدعم الوالدان الأبناء ماديا وروحيا، ويدعو المعلم الوالدين إلى المدرسة لمناقشة تربية الأبناء، ويقوم المعلم زيارة المنزل لتعريف حالات الأسرة، وتُعدّ المدرسة المنحة الدراسية لكل المتعلمين المتفوقينأكاديميا وغيرأكاديمي²6. هذا قد ذكره القائمي بأن الآباء يتولون مسؤولية مباشرة في تربية أبنائهم 27. 4. - حلول المشكلات المتعلقة بالمدرسة. ومن المعالجة لهذه المشكلات هي أن يعاشر المتعلم مع سائر الزملاء الذين لهم الأخلاق المحمودة ويجتنب عن الذين لهم الأخلاق المذمومة، وأن يستوفي المعلم

24 Al-Qaimi.

25 Nurul Afifah, 'Problematika Pendidikan di Indonesia (Telaah dari Aspek Pembelajaran)', Jurnal Elementary, 1 (2015), 41-47 (p. 44).

26 Ruhansih, p. 3.

27 Al-Qaimi, p. 390. 
كفائته كلها، ويتعامل مع المعلمين الآخرين وكذلك مع المتعلمين بخير التعامل حسب الحالة والحاجة، وينظّم المعلم البرامج والمناهج الدراسية الواضحة يستخدمها في عملية التربية والتعليم، وتحسين دور المعلم لقيام التوجياه والإرشاد عند المتعلمين. لابد للمدرسة أن تستوفي الكتب المدرسية المحتاجة لدعم عملية التربية المناسبة من خلال الشراء أو طباع تأليف المعلمين فيها أو غيره، وتمتلك المدرسة الرؤية العظيمة التي لها الأهداف العليا لنمو حماسة أهلها لتقدّم المدرساة، وتكميل الوسائل التعليمية الحديثة حسب الحاجات، وتقديم التقييم الموضيوعي العادل، وتزديد كفاءة المعلم وتطويرها مجددا. كما قد ذكره القائمي أن المدرسة مسؤولية في تربية أبناء المجتمع بشكل رسي 28. 5. لول المشكلات المتعلقة بالمجتمع.

حلول المشكلات المتعلقة بالمجتمعهي بقيام الدروس الإضافية في مراكز التعلم للمجتمع،ويشارك المجتمع في اللجان المدرسية فعالا29. 6. حلول المشكلات المتعلقة بالحقائق الاقتصادية. ومن حلولها إنماء الجهود للعمل عند الأبوين والطلبة. 7. حلول المشكلات المتعلقة بالحقائق الاجتماعية.

كما قد ذكر هداية في مقالته، أن الفجوة الاجتماعية في عصر العولمة تستطيع أن نعالجها من خلال مطالعة العلوم وجوديا ومعرفيا وإكسيولوجيا. فالمطالعة 28 Al-Qaimi, p. 390. 
الوجودية متعلقة بدور الإنسان وسلوكه المؤسس على قبضة الحياة ونمط الفكر

والنظر لدى الإنسان. والمطالعة المعرفية متعلقة باختيار العلوم اللائقة لتعليمها على

الطلبة نظارية وتطبيقية. والمطالعة الإكسيولوجية متعلقة باختبار واتحاد كل القيمة العالية أي الأخلاق والجمال في الحياة³0.

8. - حلول المشكلات المتعلقة بالحقائق السياسية.

كما قد ذكر مارديلي في مقالته، أن السياسة التربوية لها تأثيران الإجابي والسلبي.

ولعلاج المشكلات التربوية فينبغي أن نطبق نظام السياسة التربوية المتجهة إلى التغيير

المفضل أي سمي باه الحر، من خلال تمثيل الأخلاق الكريمة فتعويدها على كل الإنسان 31 ، خاصية للطلبة.

حلول المشكلات المتعلقة بالحقائق الجغرافية.

كما قد ذكرت هداياسـا في آرائها، أنّ العلاج لهذه المشكلات ينبغي أن تكون الحكومة

عادلا في اهتمام عملية التربية واستيفاء حاجاتها، مهما كانت المدارس في القرى أم في المدن232، في أماكن المنهزة أم المنغزلة.

10. حلول المشكلات المتعلقة بالنواقص ونقاط الضعف.

29 Heru Juabdin Sada, 'Peran Masyarakat Dalam Pendidikan Perspektif Pendidikan Islam', AlTadzkiyyah: Jurnal Pendidikan Islam, 8 (2017), 117-25 (p. 122).

30 Anwar Hidayat, 'Kesenjangan Sosial Terhadap Pendidikan Sebagai Pengaruh Era Globalisasi', Jurnal Justisi Hukum, 2.1 (2017), 15-25 (p. 25); Moh. Wardi, 'Problematika Pendidikan Islam Dan Solusi Alternatifnya (Perspektif Ontologis, Epistemologis Dan Aksiologis)’, Tadris, 8.1 (2013), 54-70 (p. 54).

31 Mardeli, 'Problematika Antara Politik Pendidikan Denga Perubahan Sosial Budaya Dan Upaya Solusinya', Tadrib: Jurnal Pendidikan Agama Islam, 1.2 (2015), 1-17 (p. 16).

32 Alinda Surya Hidayasa, 'Permasalahan Pendidikan Di Indonesia', UMM, 2014 <http://www.umm.ac.id/en/opini/permasalahan-pendidikan-di-indonesia.html>. 
كما قد ذكرت ميغاوانتي في مقالتها، أنّ العلاج لهذه المشكلات محتاجة إلى مشـاركة

كل نفر المرتبط بنظام التربية. وهم الآباء والمدرسون ورؤساء المدارس والمجتمع والطلبة أنفسهم. إن في مستوى المدخل، للآباء مساهمة كبيرة في تعارف القيم العالية إلى أبنائه. ولهم مسؤول كبير في تربية أبنائهم. وإن في مستوى العملية، يقوم الآباء مع المدرسين ورؤساء المدارس بتأكيد تطبيق القيم العالية لدى الأبناء كالطلبة. وإن في مستوى المخرج، ينبغي أن يستعد الطلبة لمواجهة الظواهر المتنوعاة في الحياة الحقيقية وسط المجتمع خارج المدارس باستناد إلى القيم العالية المملوكة33.

\section{منهج البحث}

واستخدم هذا البحث على منهج الدراسة الوصفية الكيفية التي تهدف إلى

توصيف ما في النصوص عميقا ونقديا 34. يعرف المنهج الوصفي على أنهالخطوات العلمية المنظمة التي توصف المشكلة أو الظاهرة، ومن ثم تحليلها بطريقة علمية واستخلاص النتائج. والمنهج النوعي أو الكيفي هي عبارة عن البحث العلمي الذي يعتمد على البيانات النوعية، حيث يُقدِّم فيه الباحث التفسيرات الشاملة لموضهوع أو مشكلة البحث العلهي، 35. فأمّا المصادر الأساسيّة هيالبحوث العلمية عن المشكلات التي تواجهها الطلبة في تعلم اللغة العربية لغير الناطقين بها. وأما المصادر الثانوية البحوث عن مشكلات

${ }^{33}$ Priarti Megawanti, 'Meretas Permasalahan Pendidikan Di Indonesia', Jurnal Formatif, 2.3 (2015), 227-34 (p. 227).

${ }^{34}$ Mahsun, Metode Penelitian Bahasa (Jakarta: Rajawali Press, 2014), p. 34.

${ }^{35}$ Mubtaits: li al-dirasatwa al istisyarat al akadimikiyah, المهج النوعي https://www.mobt3ath.com/dets.php?page 
التربية العامة وسائر النظريات المتفقة بهذا البحث. والبيانات لهذا البحث هي المشكلات التي تواجهها الطلبة ونوعية معالجتها. واستخدمت الباحثة طريقة الوثائق لجمع البيانات فيكون البحث بحثامكتبيا لأن بيانات البحث مأخوذة من الكتب والبحوث العلمية السابقة

نتائج البـحث

مشكلات التربية تواجهها الطلبة في تعلم اللغة العربية

إن المشكلات تواجهها الطلبة في المدارس الخاصة الإسلامية في الجنوب أفريقيا

وبوتسوانا تصدر من خلال المعلمين الذين يحددون تعليم اللغة العربية على أهها اللغة

لفهم القرآن ولا يحاولون على تطوير المهارات الاتصالية بها. إذان، يحدد تعليم اللغة العربية على طريقة القواعد والترجمة فقط دون الزيادة الأخرى37. ونتائج هذا البحث تدل على أن المشكلات من العقبات الخارجية المتعلقة بالمربيين. وهذه من المشكلات غير اللغوية.

وقداكتشف الدكتور الرشيد A-Rashid (2013) أن المشكلات أي الصعوبة

المواجهة لدى الطلبة في تعلم اللغة العربية لغير الناطقين بها متعلقة بمهارة الكتابة فيها أشكل الحروف الهجائية المتنوعة. ولكل الحرف نفس المخرج والصفة في النطق. ومتعلقة

${ }^{36}$ Suwardi Endraswara, Metodologi Penelitian Sastra Epistemologi, Model, Teori, dan Aplikasi (Yogyakarta: FBS Universitas Yogyakarta, 2013), p. 97.

${ }^{37}$ MA Mall and MM Nieman, 'Problems Experienced with the Teaching of Arabic to Learners in Muslim Private. Schools in South Africa and Botswana', Per Linguam: A Journal of Language Learning, 18.2 (2002), 42-54 (p. 42). 
بطريقة الكتابة المبتدأة من اليمين إلى اليسار. ومتعلقة باختلاف القواعد اللغوية بين العربية واللغة الأخرى. ومتعلقة باختلاف الثقافة العربية والثقافة لدى الطلبة.38.ونتائج هذا البحث تدل على أن المشكلات من العقبات الداخلية أي نقصان الكفاءة اللغوية عند الطلبة والعقبات الخارجية المتعلقة بالبيئة أي صعوبة المادة الدراسية. وهذه من المشكلات اللغوية.

وقد اكتشف داجاني وأخواته . Dajani, et al (2014) أن المشكلات أي الصعوبة

المواجهة لدى الطلبة في الجامعة الأردنية عند تعلم اللغة العربية لغير الناطقين بها واكتساب مهاراتها. وهي المتعلقة بخصائص المعلمين والمتعلمين، وبالبرامج التعليمية، وبجودة البرامج لإيجاد حاجات المتعلمين، وباستخدام المعمل اللغوي33.ونتائج هذا البحث تدل على أن المشكلات من العقبات الخارجية المتعلقة بالبيئة والمربيين. وهذه من المشكلات غير اللغوية.

واكتشفت العليا (2015) أن المشكلات في تعلم اللغة العربية على التلاميذ المتخرجين في المدرسة الابتدائية العامة في الفصل السابع في المدرسة المتوسطة الإسلامية الحكومية النموذجية 1 ببالنكارايا هي: الصعوبة في نطق الحروف المتقاربة مخرجا وفي التفريق بين الطويل والقصير في قراءة العربية والعثمة، واستغراق الأوقات الطويلة وكثرة التكرار لحفظ المفردات، وعدم القدرة لجعل الجملة العربية المناسبة

38 Dr. Arif Mohammed Mufleh Al-Rashid, 'Difficulties Faced by Foreign Students in Learning Arabic Language Programs for Non-Native Speakers (Evaluation Study)', Journal of Education and Practice, 4.2 (2013), 160-70 (p. 160). 
بالقواعد الصحيحة، والصعوبة في الكتابة من حيث المواءمة مع الخط، وعدم الحفز والرغبة في تعلم اللغة العربية، وعدم الوسائل التعليمية الكافية، وأساليب التعلم اللاتنوعة واللافعالية، وقليل اهتمام المعلم بهم، وكون الحصص الدراسية نهارا، وعدم الحفز والاهتمام من آبائهم في تعلمها .ونتائج هذا البحث تدل على أن المشكلات من العقبات الداخلية أي المتعلقة بنفس الطلبة والخارجية المتعلقة بالبيئة والمربيين. وهذه من المشكلات اللفوية وغير اللغوية. وقد اكتشف هارون وأخواته . Haron, et al (2016) أن المشكلات المتعلقة بتعلم الكلام العربي تصدر من العقبات الداخلية والخارجياة. فأما العقبات الداخلية تشير إلى المعوقات لدى الطلبة نفوسهم ومعرفاتهم ومهاراتهم. وأما العقبات الخارجية تشير إلى المعوقات خارج نفوس الطلبة. وهي عدم البيئة الداعمة والفرصة الكافية للتكلم العربي. وأن المشكلات التي أصابت الطلبة الملاوية معظمها العقبات الداخلية. فأما للطلبة المؤهلين تكون العقبات المتعلقة باللغة أكثر من القضايا الداخلية الأخرى. وأما للطلبة غير المؤهلين تكون العقبات المتعلقة بكثرة القصور الداخلية، كعدم الثقة واخترام الذات ${ }^{41} .{ }^{1}{ }^{2}$

${ }^{39}$ Basma Ahmad Sedki Dajani, Salwa Mubaideen, and Fatima Mohammad Amin Omari, 'Difficulties of Learning Arabic for Non-Native Speakers', Procedia - Social and Behavioral Sciences, 114.April (2014), 919-26 (p. 924).

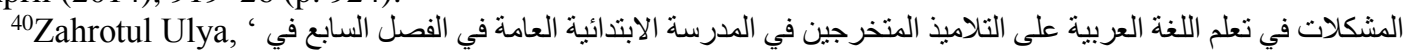
الدرسة المنوسطة الإسلامية الحكومية النموذجية 1 ببالنكار ايا 'Clalangkaraya, 2015).

${ }^{41}$ Sueraya Che Haron and others, 'Challenges in Learning to Speak Arabic', Journal of Education and Practice, 7.24 (2016), 80-85 (p. 80). 
وكان حميدي (2016) قد اكتشف أن المشكلات في تعلم اللغة العربية في الجامعة

الإسلامية بمعهد شيخنا خليل السلفي بنجكلان مادورا هي: الفروق الفردية الشخصية

لدى الطلبة، والصعوبة في تكوين البيئة اللغوية المصطنعة، والصصعوبة في تسوية المعلمين، والقصور في استخدام التكنولوجيا الحديثة 42.ونتائج هذا البحث تدل على أن المشكلات من العقبات الخارجية أي البيئة والمربيين. وهذه من المشكلات غير اللغوية. وقد وجدت فاطمة (2018) أن المشكلات في تعلم اللغة العربية لدى الطلبة الناطقين بغيرها منها: نقصان الإرادة داخل نفوس الطلبة، صعوبة قراءة النصوص العربية بل فهم معانها، نقصان الاهتمام والدافعة من آباء الطلبة عن حالة تعلمهم خارج المدرسة، طريقة الإلقاء من المعلم الواحدة أي لا يستخدم المعلم الطرق المتنوعة الفعالة، واستخدام الكتاب التعليمي الواحد فحسب بدون الزيادة من الكتب الأخرى 43.ونتائج هذا البحث تدل على أن المشكلات من العقبات الداخلية أي نقصان الكفاءة اللغوية عند الطلبة والعقبات الخارجية المتعلقة بالبيئة أي صعوبة المادة الدراسية وبالمربيين أي الآباء والمعلم. وهذه من المشكلات اللغوية وغير اللغوية. وقد اكتشف رمضان وأخواته.Romadhon, et al (2019) أن المشكلات في تعلم اللغة العربية في مدرسة نهضة العلوم بهات ياي، سونجخلا، تايلاند تنقسم إلى القسمين. فالأولى المشكلات اللغوية. هي المشكلاتالصوتيةوالمفرداتوالدلالةوالنحو والصرف

${ }^{42}$ Humaidi, 'مشكلات تعليم اللغة العربية في الجامعة الإسلامية بمعه شيخنا محمد خليل السلفي بنجكلان مادورا' (UIN Maliki Malang, 2016).

${ }^{43}$ Veti Nur Fatimah, 'Analisis Kesulitan Belajar Bahasa Arab Siswa Kelas Ix Madrasah Tsanawiyah Negeri 2 Sleman Yogyakarta’ (UII Yogyakarta, 2018), p. xvii. 
والكتابة. والثانية المشكلات غيراللغوية. هي متعلقة بالأمور الدافعة إلهها، مثل البيئة

اللغوية، الأدوات المدرسية،وحالة النفس مثل الحماسةوالنشاطة والحفز وغيرهاب. ونتائج هذا البحث تدل على أن المشكلات من العقبات الداخلية المتعلقة بنفس الطلبة والعقبات الخارجية المتعلقة بالبيئة. وهذه من المشكلات اللغوية وغير اللغوية. و لوبيس وأخواتها (2019) اكتشفوا أن المشكلات في تعلم اللغة العربية بمهارة القراءة لدى الطلبة مدرسة المتوسطة الإسلامية الحكومية الأولى بادانج سيدامبوانج هي قليل الفهم للغة العربية، وملل الطلبة، وعدم البنية التحية للمدرسية،وعدم وحدة النية بين الطلبة والآباء والمعلمين 45. ونتائج هذا البحث تدل على أن المشكلات من العقبات الداخلية المتعلقة بنفس الطلبة والعقبات الخارجية المتعلقة بالبيئة والمبربين. وهذه من المشكلات اللغوية وغير اللغوية. وإن المشكلات في تعلم اللغة العربية لدى الطلبة المتخرجين في المدارس العامة في الصف العاشر بالمدرسة الثانوية الإسلامية الحكومية الأولى هولو سونجاي الوسطى التي اكتشفتها الحسنة (2019) هي: قلة استعاب المفردات وقلة الفهم في قواعد اللغة. وهذه بسبب العوامل الداخلية أي لايريد الطلبة وفاء الوظيفات من المعلم إلا في وقت محدد وحالة محددة، وعدم الاهتمام منهم لأثناء عملية التعلم. وبسبب اللعوامل الخارجية أي

${ }^{44}$ Ilham Fatkhu Romadhon, M. Alfin Khoirun Na'im, and Yuli Agustina, 'Linguistic and NonLinguistic Problems and Their Solution to Learning Arabic at Songserm Wittaya Mulnithi School', in The 3rd International Conference of Arabic Language and Literature (Bandung: Indonesian University of Education, 2019), pp. 51-69 (p. 51).

${ }^{45}$ Desi Haryanti Lubis, Akhiril Pane, and Ismail Baharuddin, 'مشكلات تعلم اللغة العربية بمهارة القراءة), Thariqah Ilmiyah: Jurnal Ilmu-IIlmu Kependidikan \& Bahasa Arab, 7.1 (2019), 1-18 (p. 17). 
عدم الاهتمام من آبائهم وممن حولهم في تعلم اللغة العربية".46. ونتائج هذا البحث تدل على أن هذه من المشكلات اللغوية وغير اللغوية. إن المشكلات التي تواجهها الطلبة في تعلم قراءة النصوص العربية خاصة القرآن الكريم هي المتعلقة بالصعوبة من ناحية الصوتي والصرفي والتركيبي والدلالي 47. ونتائج هذا البحث تدل على أن المشكلات من العقبات الداخلية أي المتعلقة بنفس الطلبة وهي ضعف الكفاءة اللغوية العربية. وكذلك العقبات الخارجية المتعلقة بالبيئة أي صعوبة المادة الدراسية. وهذه من المشكلات اللغوية.

والمشكلات التي تواجهها الطلبة في تعلم قراءة الكتب العربية التي اكتشفتها سورياني ودولائي (2019) هي: الصعوبةفي تعلم صيغة الكلمة، وعدم المعرفة عن إعراب الجمل، الصعوبة في تحديد الخطوط، والصعوبة في فهم الكلمات في النصوص، عدم الحفز في التعلم، نقصان الطريقة التعليمية المتنوعة من المعلم، ونقصان المراجعة، وعملية التعليم مملة، ونقصان الحصص الدراسية 48. ونتائج هذا البحث تدل على أن المشكلات من العقبات الداخلية أي المتعلقة بنفس الطلبة وهي ضعف الكفاءة اللغوية العربية. وكذلك العقبات الخارجية المتعلقة بالبيئة وبالمربيين. وهذه من المشكلات

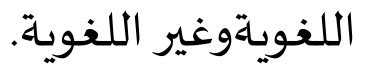

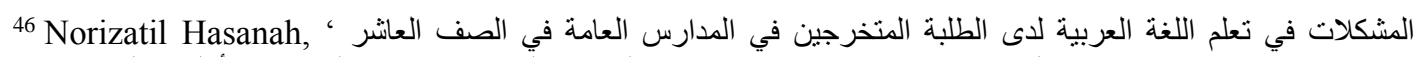

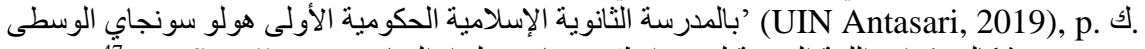

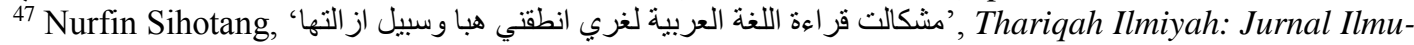
IIlmu Kependidikan \& Bahasa Arab, 7.1 (2019), 1-16 (p. 1).

${ }^{48}$ Wilda Suryani and Abdul Sattar Daulay, 'مشكلات تعليم قر اءة الكتب لطلبة قسم تدريس اللغة العربية', Thariqah Ilmiyah: Jurnal Ilmu-IIlmu Kependidikan \& Bahasa Arab, 7.1 (2019), 1-17 (p. 1).
} 


\section{حلول مشكلات التربية تواجهها الطلبة في تعلم اللغة العربية}

قد قدم عصام الدين الحل العاملعلاج المشكلات السابقة، منه: يحاول المعلم

استخدام الأسـاليب والإستراتيجيات التي تشجع الطلبة في تعلم اللغة العربية، تؤسس المدرسة البرنامج الخاص لتعليم اللغة العربية، تبني مدرسة بيئة اللغة العربية، ويعطي المعلم الطلبةحفظ المفردات الفعالية لتعليم اللغة العربية.49. وقد قدم الأعظمي الحلول لعلاج المشكلات اللغوية في تعليم اللغة العربية لغير الناطقين بها، منها: توجياء الطلبة إلى الاستفادة من وسائل الفصحى خارج الفصل، توثيق الصلة بين الطلبة والبيئة المثقفة العربية، تحسين بيئة المدرسة الملائمة للغة العربية، تعيين الأساتيذ المؤهلين المتخصصين في اللغة العربية، تنشيط أذهان الطلبة وتشويقهم إلى اللغة العربية بالتقنيات والوسائل الحديثة.50وقد ذكر عقيقي الحل الآخر لعلاج المشكلات السابقة، خاصة من خلال اختيار المواد التعليمية المناسبة بكفاءة الطلبة المملوكة

وقد ذُكر في كتاب قراءة في صعوبات تعلهم مادة اللغة العببية في العراق، أن

الحل لبعض المشكلات السابقة تستطيع معالجتها بالوسائل لتسهيل تعليم مادة اللغة العربية. منها: تدريب المعلم على المهارات والطرائق المناسبة في تدريس اللغة العربية، إعادة

\footnotetext{
${ }^{49}$ M. Ishomuddin, 'مشكلات تعليم اللغة العربية في مدرسة مفتاح العلوم الثانوية بكالسات جمبر وحلها' (UIN Maliki Malang, 2015), p. J.

صعوبات تعلم اللغة العربية للناطقين بغير ها: الأسباب وطرق ووسائل ، صوائل

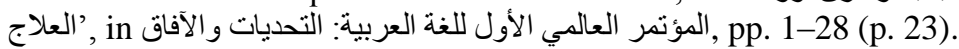

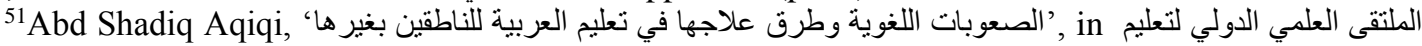
(Mesir: Al Sheikh Zayed Center for Teaching Arabic for Non Natives, 2013).
} 
ترتيب تسلسل موضيوعات مادة اللغة العربية، تدريب الطلبة على المهارات اللغوية العربية، ، إقامة المسابقة للطلبـة بالموضوعات الخاصية باللغة العربية وفروعهام2.

وقد ذكر في البحث الجامعي للزهراء أن من الحلول لمعالجـة المشكلات في تعلم

اللغة العربية للناطقين بغيرها أي الصعوبات فياه هي: تزويد الحصص الدراسية، وتكميل الوسائل التعليمية في المعمل اللغوي، وأداء التعامل مع الأسـاتيذ الأخرى لأن ينظم البيئة اللغوياة العربية، خاصية في المعهد أو السكن للطلبـة. هذه الحلول التي يستطيع أن يعملها المعلم. أما الحلول التي يستطيع أن يعملها الطلبة هي إنماء الموهوب والإرادة لفهم اللغة العربية، وممارسـة المهارات اللغوية الأربعة، وينبغي أن يمتلك الطلبة التعيين لحفظ المفردات الجديدة أي زيادتهات53. - ت

وقد ذكر أرديانشـاه في بحثـا الجامعي أن من الحلول لمعالجـة المشكلات في تعلم

اللغة العربية للناطقين بغيرها أي الصعوبات فيه هي: أداء المناقشاة عن المواد غير المفهومة عند الطلبة،، إصناع الطرق المتنوعاة لفهم المواد الدراسية، إعطاء النصيحة للطلبة كي يتعلموا مع زملاءهم العُلمى منهم، إعطاء الحفز للطلبة كي يتحمّسـوا لتعلم اللغة العربية داخل المدرسة وخارجها، والمحاولة لمسح ظن الطلبة عن صعوبة اللغة العربية.54.

${ }^{52}$ Majelis el-Nuwab el-Iraqi, pp. 7-8.

${ }^{53}$ Fatimah Azzahra, 'Upaya Dalam Mengatasi Kendala Pembelajaran Bahasa Arab Bagi Siswa Lulusan SMP Di MA Islamiyah Nahdlatuttullab (MA MINAT) Kesugihan Cilacap' (UIN Sunan Kalijaga Yogyakarta, 2015), p. ix.

${ }^{54}$ Muhammad Beben Satri Ardiansyah, 'Analisis Faktor Kesulitan Membaca Teks Berbahasa Arab Pada Siswa Kelas X IS 1 MAN Wonokromo Bantul Yogyakarta Tahun Ajaran 2016/2017’ (UIN Sunan Kalijaga, 2017), p. xiii. 
وقد ذكر ناور في بحثه الماجستير أن هناك محتاج إلى الاهتمام الخاص للطلبة

المتخرجين في المدارس العامة من خلال الإجراءات التعليمية لدى المعلمين، وإلى الأنشطة اللغوية العربية الجودية من خلال جمعيات الطلبة العربية كي تسعاعدهم في تنظيم البيئة اللغوية العربية

وقد ذكر ستياوان أن من القوانين المتعلقة بالبرنامج التعليمي لتوحيد جميع الطلبة الذين لهم اختلاف الخلفية التربوية هي: تقسيم أعضاء الفصل حسب كفاءة الطلبة، أداء الحصة الزيادة للطلبة لهم الكفاءة الضعيفة عن المهارات اللغوية العربية. ومن الطرق التي يستطيع أن يعملها الطلبة لترقية مهاراتهم اللغوية العربية هي: حفظ المفردات، أداء زيادة التعلم مستقلا، مشاركة في حلقة التعليم للغة العربية مع المتدربين من نفس المرحلة، مشاركة في برنامج تطوير اللغة العربية في الجامعة، ومشاركة في المقرر لتعلم اللغة العربية خارج الجامعة.56.

الخلاصة

إن المشكلات التي تواجهها الطلبة أثناء تعلم اللغة العربية لغير الناطقين بهاتصدر من العقبات الداخلية والخارجية. فالعقبات الداخلية متعلقة بنفس الطلبة. منها الحالة النفسية والكفاءة اللغوية لديهم. والعقبات الخارجية متعلقة بالبيئة والمربيين.منهاصعوبة

${ }^{55}$ Andi Rahmat Nawir, 'Analisis Kesulitan Belajar Bahasa Arab Mahasiswa Alumni Sekolah Umum Pada Jurusan Pendidikan Bahasa Arab Fakultas Tarbiyah UIN Alauddin Makassar' (UIN Alauddin Makassar, 2014). 
اللغة العربية مهاراتها ونظامها لدى الطلبة بغير الناطقين بها، وعدم البيئة اللغوية الجودية، وضعف جهود المعلمين، ونقصان الوسائل التعليمية الفعالة.إن المشكلات غير اللغوية أكثر من المشكلات اللغوية. وهذه المشكلات لا بد من حلولها. ومنها لا بد للمتعلم أن يكون بتزكية النفس ويعلم أهمة العلوم، ويحفظ المفردات، ويزيد التعلم مستقلا، ومشاركة في حلقة التعليم مع المتدربين من نفس المرحلة، ومشاركة في برنامج تطوير اللغة العربية. ولا بد للمعلم أن يحاول في استخدام الأساليب والإستراتيجيات التي تشجع الطلبة في التعلم، وأن يدرب على المهارات والطرائق المناسبة في تدريس اللغة العربية. وأداء المناقشة عن المواد غير المفهومة عند الطلبة، وينصح الطلبة كي يتعلموا مع زملائهم ويحفزهم لحماستهم في التعلم، والمحاولة لمسح ظن الطلبة عن صعوبة تعلم اللغة العربية. وعلى الأسرة لا بد أن تختار المدرسة اللائقة بالأولاد وتهتم بتربيتهم، وعلى المدرسة أن تستوفي مايحتاجه المتعلم من المواد والمعلم المأهل والبيئة ودروس إضافية وإقامة المسابقة للطلبة والأنشطة اللغوية العربية الجودية وإنماء الموهوب والإرادة لفهم اللغة العربية، والاهتمام الخاص للطلبة المتخلفين.

الشكروالتنويه

الحمدلله رب العالمين، لايطيب الليل إلا بشكرك، ولايطيب الهار إلى بطاعتك وذكرك، والشكر إلى من بلغ الرسالة وأدى الأمانة ونصح الأمة سيدنا محمد صلى الله عليه

${ }^{56}$ Agung Setiyawan, 'Problematika Keragaman Latar Belakang Pendidikan Mahasiswa Dan Kebijakan Program Pembelajaran Bahasa Arab', Arabiyat: Jurnal Pendidikan Bahasa Arab Dan 
وسلم. أقدم أسمى شكرنا وتقديرنا إلى والدي الذي علمني العطاء بدون انتظار ووالدتي التي هي بسمة الحياة وسر الوجود، وسر نجاحي بدعائها. وكذلك أشكر كل من ساعد على إتمام هذا البحث وقدم لي العون ومد لي يد المساعدة وزودني بالمعلومات اللازمة.كما لا أنسى أن أقدم شكري العميق لجامعة مالانج الحكومية (Universitas Negeri Malang) التي أستفيد منها العلم والمعرفة وفيها أعمل لخدمة اللغة العربية.

Afifah, Nurul, 'Problematika Pendidikan di Indonesia (Telaah dari Aspek Pembelajaran)', Jurnal Elementary, 1 (2015), 41-47

Al-A'dhami, Mahmud Ya'qub Al-Hindi, ' صعوبات تعلم اللغة العربية للناطقين بغير ها: الأسباب وطرق

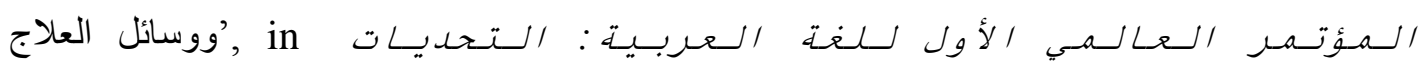
قو / و الافـ pp. 1-28

Al-Anzi, Saud Farhan, ، المشكلات التي تواجه طلبة التربية العملية في جامعة شقراء من وجهة نظر الطلبة 'المعلمين أنفسهر , Journal of University of Babylon for Humanities, 23 (2015), 3-21

Al-Awami, Abdullah Ali, and Ahmad Yahya Al-Kuri, 'المشكلات التي يواجها طلبة الدبلوم المهني بجامعة العين للعلوم و التكنولوجيا في التربية العملية الميدانية , Journal of Al-Nasser University, 8 (2016), 196-225

Al-Bashtawi, Abeer Hameed, 'Student's Perspectives for the Encountered Problems \& Challenges at the Department of English in The Colege of Sciences \& Arts In Alqurrayyat', International Journal of Education and Research, 2.2 (2014), 1-8

Al-Ma'jal, Thilal bin Muhammad, and Ibrahim Abdulaziz Mega, ، مشكلات تدريس التربية 'الإسلامية في المرحلة المتوسطة بالمدارس العربية الإسلامية في مالي من وجهة نظر المعلمين, IUGJEPS, 25.2 (2017), 82-99

Al-Qaimi, Ali, أسس الـتــبـيـة (Beirut: Daar el-Nubala, 1995)

Al-Rashid, Dr. Arif Mohammed Mufleh, 'Difficulties Faced by Foreign Students in Learning Arabic Language Programs for Non-Native Speakers (Evaluation Study)', Journal of Education and Practice, 4.2 (2013), 160-70

Kebahasaaraban, 5.2 (2018), 195-213 (p. 211). 
Al-Salakhi, Mahmoed Gamal Gamil, ' مشكلات التربية العملية من جهة نظر الطالبات المعلمات في جامعة البترا الخاصة', Majalah Mutah li al-Buhuts wa al-Dirasat: Silsilah al-Ulum alInsaniyah wa al-Ijtima 'iyah, 25.7 (2010), 199-234

Al-Subyani, Noor Abdulhadi, 'The Academic, Administrative, Economic, Social, and Psychological Problems Faced by Students of Textile and Clothing Major at King Abdul-Aziz University', International Education Studies, 2016, 155-62

Al-Syuhubi, Hasan Salim, and Ibrahim Otsman Arhim, ' المشكلات التي تو اجه الطلبة المعلمين أثناء 'ممارستهم للتربية العملية من وجهة نظر الطلبة أنفسهم , Journal of Education Faculty Misurata University, 5 (2016), 184-208

Anderson, D, 'Geographic and Economic Factors and Developmet of Educational System in Western Europe', The University of Chicago Journals, 9.2 (1965), 14754

Aqiqi, Abd Shadiq, 'الصعوبات اللغوية وطرق علاجها في تعليم العربية للناطقين بغيرها', in الـملـتــى

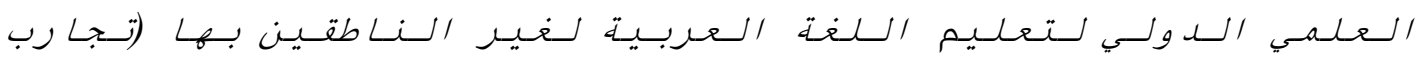
و ورؤي مستقـبــيـة (Mesir: Al Sheikh Zayed Center for Teaching Arabic for Non Natives, 2013)

Ardiansyah, Muhammad Beben Satri, 'Analisis Faktor Kesulitan Membaca Teks Berbahasa Arab Pada Siswa Kelas X IS 1 MAN Wonokromo Bantul Yogyakarta Tahun Ajaran 2016/2017' (UIN Sunan Kalijaga, 2017)

Azzahra, Fatimah, 'Upaya Dalam Mengatasi Kendala Pembelajaran Bahasa Arab Bagi Siswa Lulusan SMP Di MA Islamiyah Nahdlatuttullab (MA MINAT) Kesugihan Cilacap' (UIN Sunan Kalijaga Yogyakarta, 2015)

Dajani, Basma Ahmad Sedki, Salwa Mubaideen, and Fatima Mohammad Amin Omari, 'Difficulties of Learning Arabic for Non-Native Speakers', Procedia - Social and Behavioral Sciences, 114.April (2014), 919-26

Doygun, Ozlem, and Selma Gulec, 'The Problem Faced by University Students and Proposal for Solution', 2012, XLVII, 1115-23

Endraswara, Suwardi, Metodologi Penelitian Sastra Epistemologi, Model, Teori, dan Aplikasi (Yogyakarta: FBS Universitas Yogyakarta, 2013)

Fahrurrozi, Aziz, 'Pembelajaran Bahasa Arab: Problematika Dan Solusinya', ARABIYAT: Jurnal Pendidikan Bahasa Arab Dan Kebahasaaraban, 1.2 (2014), 161-80 <https://doi.org/10.15408/a.v1i2.1137>

Fatimah, Veti Nur, 'Analisis Kesulitan Belajar Bahasa Arab Siswa Kelas Ix Madrasah 
Tsanawiyah Negeri 2 Sleman Yogyakarta' (UII Yogyakarta, 2018)

Haron, Sueraya Che, Ismaiel Hassanien Ahmed, Arifin Mamat, Wan Rusli Wan Ahmad, and Fouad Mahmoud M. Rawash, 'Challenges in Learning to Speak Arabic', Journal of Education and Practice, 7.24 (2016), 80-85

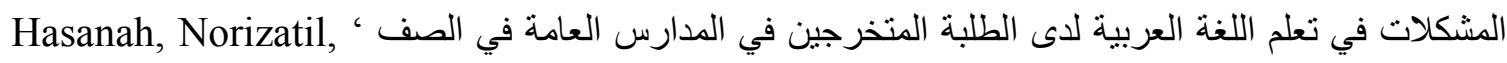
(العاثر بالمدرسة الثانوية الإسلامية الحكومية الأولى هولو سونجاي الوسطى (UIN Antasari, 2019)

Hidayasa, Alinda Surya, 'Permasalahan Pendidikan Di Indonesia', UMM, 2014 <http://www.umm.ac.id/en/opini/permasalahan-pendidikan-di-indonesia.html>

Hidayat, Anwar, 'Kesenjangan Sosial Terhadap Pendidikan Sebagai Pengaruh Era Globalisasi', Jurnal Justisi Hukum, 2.1 (2017), 15-25

Humaidi, 'مشكلات تعليم اللغة العربية في الجامعة الإسلامية بمعهد شيخنا محمد خليل السلفي بنجكلان مادورا' (UIN Maliki Malang, 2016)

Ishomuddin, M., 'مشكلات تعليم اللغة العربية في مدرسة مفتاح العلوم الثانوية بكالسات جمبر وحلها' (UIN Maliki Malang, 2015)

Ismah, and Erna Ratna Wibiastuti, 'Pengaruh Letak Geografis Sekolah Terhadap Konsentrasi Belajar Matematika Sekolah Menengah Pertama', FIBONACCI: Jurnal Pendidikan Matematika \& Matematika, 1.1 (2015), 82-96

Istiqomah, Himatul, and Halimi, ، اتجاهات كون النبي والرسول في شخصية محمد في نثر مولد 'البرزنجي ,'Arabi: Journal of Arabic Studies, 1, 2 (2017), 127-40

Khofifah, Aulia, Afrizal Sano, and Yarmis Syukur, 'Permasalahan yang Disampaikan Siswa kepada Guru BK/Konselor', Jurnal Educatio; Jurnal Pendidikan Indonesia, 3.1 (2017), 45-52

Khuwalda, Mushthofa Fankhur, Fathi Mahmoed Ahameeda, and Saad Abdul Qadir AlHijazi, ، مشكلات التربية العملية التي تو اجه الطلبة المعلمين في تخصص تربية طفل في كلية الملكة رانيا للطفولة بالجامعة الهاثمية', Journal of Damascus University, 26.3 (2010), 737-81

Lubis, Desi Haryanti, Akhiril Pane, and Ismail Baharuddin, “ مشكلات نعلم اللغة العربية بمهارة القراءة', Thariqah Ilmiyah: Jurnal Ilmu-IIlmu Kependidikan \& Bahasa Arab, 7.1 (2019), 1-18

Mahsun, Metode Penelitian Bahasa (Jakarta: Rajawali Press, 2014)

Majelis el-Nuwab el-Iraqi, 'قراءة في صعوبات تعلم مادة اللغة العربية في العراق', in اقسـ ورة الانـتخا بـية الستـانـيـة (Iraq: Dairah el-Buhuts, 2014)

Mall, MA, and MM Nieman, 'Problems Experienced with the Teaching of Arabic to Learners in Muslim Private ${ }_{3}$ Schools in South Africa and Botswana', Per Linguam: 
A Journal of Language Learning, 18.2 (2002), 42-54

Mardeli, 'Problematika Antara Politik Pendidikan Denga Perubahan Sosial Budaya Dan Upaya Solusinya', Tadrib: Jurnal Pendidikan Agama Islam, 1.2 (2015), 1-17

Megawanti, Priarti, 'Meretas Permasalahan Pendidikan Di Indonesia', Jurnal Formatif, 2.3 (2015), 227-34

Microsoft @ Encarta. Languages Spoken by More Than 10 Million People (2006)

Mubtaits: li al-dirasatwa al istisyarat al akadimikiyah, المهجج

النوعيhthtps://www.mobt3ath.com/dets.php?page

Nawir, Andi Rahmat, 'Analisis Kesulitan Belajar Bahasa Arab Mahasiswa Alumni Sekolah Umum Pada Jurusan Pendidikan Bahasa Arab Fakultas Tarbiyah UIN Alauddin Makassar' (UIN Alauddin Makassar, 2014)

Permadi, Nofianti Eka, 'Masalah-Masalah yang Dihadai Peserta Didik dalam Perencanaan Karir dan Implikasinya Terhadap Pelayanan Bimbingan Karir’, Jurnal Penelitian Bimbingan dan Konseling, 2, 1 (2016), 134-45

Romadhon, Ilham Fatkhu, M. Alfin Khoirun Na'im, and Yuli Agustina, 'Linguistic and Non-Linguistic Problems and Their Solution to Learning Arabic at Songserm Wittaya Mulnithi School', in The 3rd International Conference of Arabic Language and Literature (Bandung: Indonesian University of Education, 2019), pp. 51-69

Ruhansih, Dea Siti, 'Masalah-Masalah Siswa Di Sekolah Serta Pendekatan-Pendekatan Umum Dalam Bimbingan Dan Konseling Ditinjau Dari “Kacamata Konselor (Studi Deskriptif Di SMP Nugraha Bandung)', Jurnal Quanta, 2.1 (2018), 1-3

Sada, Heru Juabdin, 'Peran Masyarakat Dalam Pendidikan Perspektif Pendidikan Islam', Al-Tadzkiyyah: Jurnal Pendidikan Islam, 8 (2017), 117-25

Setiyawan, Agung, 'Problematika Keragaman Latar Belakang Pendidikan Mahasiswa Dan Kebijakan Program Pembelajaran Bahasa Arab', Arabiyat: Jurnal Pendidikan Bahasa Arab Dan Kebahasaaraban, 5.2 (2018), 195-213

Sihotang, Nurfin, 'مشكالت قراءة اللغة العربية لغري انطقني هبا وسبيل از التها', Thariqah Ilmiyah: Jurnal Ilmu-IIlmu Kependidikan \& Bahasa Arab, 7.1 (2019), 1-16

Suryani, Wilda, and Abdul Sattar Daulay, 'مشكلات تعليم قر اءة الكتب لطلبة قسم تدريس اللغة العربية', Thariqah Ilmiyah: Jurnal Ilmu-IIlmu Kependidikan \& Bahasa Arab, 7.1 (2019), 117

Syafini, Elgi, Yarmis Syukur, and Indra Ibrahim, 'Masalah Belajar Siswa dan Penanganannya', Konselor; Jurnal Ilmiah Konseling, 2.2 (2013), 15-19 
Ulya, Zahrotul, ' المشكلات في تعلم اللغة العربية على التلاميذ المتخرجين في المدرسة الابتدائية العامة في (الفصل السابع في المدرسة المنوسطة الإسلامية الحكومية النموذجية 1 ببالنكارايا (IAIN Palangkaraya, 2015)

Wardi, Moh., 'Problematika Pendidikan Islam Dan Solusi Alternatifnya (Perspektif Ontologis, Epistemologis Dan Aksiologis)', Tadris, 8.1 (2013), 54-70 